\title{
Cholesterol Enhances Colorectal Cancer Progression via ROS Elevation and MAPK Signaling Pathway Activation
}

\author{
Caihua Wang ${ }^{\mathrm{a}}$ Peiwei Li Junmei Xuan ${ }^{\mathrm{b}}$ Chunpeng Zhu ${ }^{\mathrm{a}}$ Jingjing Liu ${ }^{\mathrm{b}}$ Lizhen Shan $^{\mathrm{b}}$ \\ Qin Du ${ }^{a}$ Yuezhong Ren ${ }^{b}$ Jun Ye ${ }^{a}$ \\ aDepartment of Gastroenterology, The Second Affiliated Hospital, Zhejiang University School of \\ Medicine, Hangzhou, 'Department of Endocrinology and Metabolism, The Second Affiliated Hospital, \\ Zhejiang University School of Medicine, Hangzhou, China
}

Key Words

Cholesterol $\bullet$ Colorectal cancer $\bullet$ ROS $\bullet$ Metastasis

\begin{abstract}
Background/Aims: Elevated serum cholesterol levels were linked to a higher risk of colorectal adenoma and colorectal cancer (CRC), while the effect of cholesterol on CRC metastasis has not been widely studied. Methods: CRC patients were enrolled to evaluate the association between low-density lipoprotein cholesterol $(L D L)$ and CRC metastases, and LDL receptor (LDLR) level of the CRC tissue was assessed by immunohistochemistry. The effects of LDL on cell proliferation, migration and stemness were assessed in CRC cells in vitro, and the effects of high fat diet (HFD) on tumor growth and intestinal tumorigenicity were investigated in vivo. ROS assays, gene expression array analysis and western blot were used to explore the mechanisms of LDL in CRC progression. Results: The level of LDL was positively correlated with liver metastases, and a higher level of LDL receptor (LDLR) expression was associated with advanced $\mathrm{N}$ and $\mathrm{M}$ stages of CRC. In vitro, LDL promoted the migration and sphere formation of CRC cells and induced upregulated expression of "stemness" genes including Sox2, Oct4, Nanog and Bmi 1. High-fat diet (HFD) significantly enhanced tumor growth in vivo, and was associated with a shorter intestinal length in azoxymethane/dextran sodium sulfate (AOM/DSS)-treated mice. Furthermore, LDL significantly elevated reactive oxygen species (ROS) levels and Whole Human Genome Microarray found 87 differentially expressed genes between LDL-treated CRC cells and controls, which were largely clustered in the MAP kinase (MAPK) signaling pathway. Conclusions: LDL enhances intestinal inflammation and CRC progression via activation of ROS and signaling pathways including the MAPK pathway. Inflammation is strongly associated with cancer initiation, and the role of LDL in intestinal tumorigenicity should be further explored.




\section{Introduction}

Colorectal cancer (CRC) remains one of the most common types of malignancy worldwide, and CRC-related mortality is largely caused by metastasis $[1,2]$. Individuals with obesity are at a higher risk of certain types of malignancy, including CRC. As premalignant lesions, colorectal adenomas exhibit multiple molecular pathway variations and can progress into invasive cancer via the conventional adenoma-carcinoma sequence [3]. Previous studies have demonstrated an association between serum lipids and the risk of colorectal adenomas, indicating a possible role of serum lipids in cancers of the gastrointestinal (GI) tract [4]. Moreover, cholesterol has been observed to accumulate generally in various malignant tissues, and some studies have also suggested that cholesterol plays an important role in the development of cancer [5, 6]. However, some other studies have reported decreased serum lipid levels in cancer patients at advanced stages, possibly due to poor nutrition status [7]. Thus the association between cholesterol and cancer needs further research. In addition, only a few studies have investigated the association between serum cholesterol levels and the progression of CRC; therefore, the conclusion remains unclear.

The mammalian intestine can respond to dietary signals. It is increasingly being recognized that diet is a significant risk factor in cancer initiation and progression in multiple tumor types. One mechanism by which this occurs is via biological changes in adult stem cells $[8,9]$. A high-fat diet (HFD) was shown to induce peroxisome proliferatoractivated receptor delta (PPAR- $\delta$ ) expression in intestinal stem cells and progenitor cells, indicating that HFD could augment the capacity of intestinal stem and progenitor cells to initiate tumors [10]. Additionally, another study indicated that the number of colonic green fluorescent protein-positive (GFP+) stem cells significantly increased in HFD mice compared with controls. Increased cell proliferation and decreased apoptosis were also observed in Lgr5(+) stem cells in response to injection with azoxymethane (AOM), suggesting that HFDinduced obesity can promote colonic stem cell expansion during cancer initiation [11]. Stem cells are undifferentiated cells that can differentiate into specialized cells, while cancer stem cells may generate tumors through self-renewal and differentiation into multiple cell types. Cancer stem cells may play a vital role in cancer relapse and metastasis, due to their proliferative potential and strong resistance to therapy [12].

Studies have also suggested that HFD may contribute to inflammation and oxidative stress by causing excessive production of reactive oxygen species (ROS) [13]; moreover, both LDL and VLDL were shown to activate the extracellular signal-regulated kinase (ERK)/ mitogen-activated protein kinase (MAPK) pathway [14]. ROS are a group of molecules and free radicals generated within cells through oxygen metabolism. Excessive ROS production can result in oxidative stress, characterized by oxidative damage to proteins, lipids and DNA [15]. Evidence has indicated an important role for ROS induction in cancer development, metastasis, progression, and survival [16]. The effects of ROS might be associated with multiple signaling pathways, including the MAPK pathway, which is one of the principal signaling conduits controlling cell proliferation and differentiation [15]. Moreover, ROS and the MAPK pathway also play vital roles in inflammation, which is strongly associated with cancer initiation [17].

Considering the current evidence of the association between cholesterol and CRC, and the effects of cholesterol on ROS and MAPK pathway activation, we hypothesize that cholesterol enhances the tumorigenicity of intestinal cells and CRC progression via ROS and the MAPK pathway. To test this hypothesis, we performed cohort studies comprising CRC patients to explore the effects of cholesterol and the LDL receptor (LDLR) on cancer progression, and in vivo and in vitro experiments to assess the effects of LDL on CRC tumorigenicity, growth, migration and stemness. Furthermore, the association between LDL and ROS/the MAPK pathway was also evaluated by DCFH-DA fluorescence analysis and Whole Human Genome Microarray.

\section{KARGER}




\section{Cellular Physiology Cell Physiol Biochem 2017;42:729-742 \begin{tabular}{l|l} 
and Biochemistry Published onlıne: June 09, 2017 & $\begin{array}{l}\text { (c) } 2017 \text { The Author(s). Published by S. Karger AG, Basel } \\
\text { www.karger.com/cpb }\end{array}$
\end{tabular}}

\section{Materials and Methods}

\section{Ethics statement}

The current research was approved by the Institutional Review Board of the Second Affiliated Hospital of Zhejiang University School of Medicine. Informed consent was obtained from all participants for the use of the clinical information in this research. All experiments involving animals in this study were approved by Institutional Review Board of the Second Affiliated Hospital of Zhejiang University School of Medicine, and were conducted in accordance with relevant national and international guidelines and regulations.

\section{Patient enrollment, follow-up, and tissue array}

A total of 90 patients who underwent surgery for histologically proven colorectal cancer (CRC) at hospitals that cooperated with the National Engineering Center for Biochip at Shanghai, China, during the period from April 2008 to November 2008, were selected for this research. Paraffin-embedded tumor specimens and paired adjacent non-tumor specimens $(\leq 1.5 \mathrm{~cm}$ away from the tumor) were carefully collected before any treatment was administered. The formalin-fixed, paraffin-embedded colorectal cancer tissue samples that were collected were reassembled into multiple tissue arrays. All patients were followed up until September 2014. Patients were provided with written information regarding the course and purpose of the study, and all provided written consent to participate, prior to being included in the study. In addition, we also evaluated another cohort enrolling 41 CRC patients with liver metastases and 50 CRC patients without metastases, to evaluate the association between lipid profiles and metastasis of CRC. All those 91 CRC cases were newly diagnosed between May 2014 and September 2015 at the Second Affiliated Hospital of Zhejiang University School of Medicine (Zhejiang, China). The diagnosis of CRC for each case was confirmed independently by two pathologists. Clinicopathological characteristics of the CRC patients in our study are presented in Table 1.

\section{Cell lines and cell culture}

The human colorectal cancer cell lines SW480, LoVo and RKO were purchased from the cell bank at the China Academy of Medical Science (China). These cells were cultured in RPMI-1640 medium (Gibco, Carlsbad, CA, USA) containing $10 \%$ fetal bovine serum (FBS, Gibco) and maintained at $37^{\circ} \mathrm{C}$ in a humidified 5\% $\mathrm{CO}_{2}$ atmosphere. LDL (Meilun Biology Technology, Dalian, China) was added to medium at a concentration of $100 \mu \mathrm{g} / \mathrm{ml}$ to assess its influence on cell proliferation, migration and sphere formation compared with controls. The concentration of LDL was determined by preliminary experiment comparing LDL concentrations of $50 \mu \mathrm{g} / \mathrm{ml}, 100 \mu \mathrm{g} / \mathrm{ml}$ and $200 \mu \mathrm{g} / \mathrm{ml}$, and the results indicate that a concentration of $100 \mu \mathrm{g} / \mathrm{ml}$ was superior to $50 \mu \mathrm{g} / \mathrm{ml}$ but similar to $200 \mu \mathrm{g} / \mathrm{ml}$.

\section{Cell proliferation}

SW480, LoVo or RKO cells were seeded at a density of $1 \times 10^{3}$ cells per well in a 96 -well plate containing $200 \mu \mathrm{l}$ RPMI-1640 medium with 10\% FBS. 3-[4, 5-dimethylthiazol-2-yl]-5-[3-carboxymethoxy-phenyl]-2[4-sulfophenyl]-2H-tetrazolium salt reagent (MTS) (Promega, Madison, WI, USA) (20 $\mu$ l) was added to each well and the cells were incubated at $37^{\circ} \mathrm{C}$ for $4 \mathrm{~h}$. The absorbance values were measured at $490 \mathrm{~nm}$ on a microplate reader (Bio-Rad, Hercules, CA, USA) and assessed continuously for 5 days.

\section{Cell migration assay}

Transwell insert chambers with $8-\mu$ m porous membranes (Corning Incorporated, NY, USA) were used for motility assays. LoVo or RKO cells were placed in the top chamber at a density of $5 \times 10^{4}$ cells per well, in serum-free media. RPMI-1640 medium containing 15\% FBS was added to the bottom chamber. Cells were incubated for $24 \mathrm{~h}$ at $37^{\circ} \mathrm{C}$ in $5 \% \mathrm{CO}_{2}$. To quantify migrating cells, cells were removed from the upper surface,

Table 1. Characteristics of the included CRC patients. All continuous variables are expressed as mean \pm standard deviation (SD). LDL, low-density lipoprotein; HDL, high-density lipoprotein

\begin{tabular}{llll}
\hline Characteristics & $\begin{array}{l}\text { Patients with liver } \\
\text { metastasis }\end{array}$ & $\begin{array}{l}\text { Patients without liver } \\
\text { metastasis }\end{array}$ & P value \\
\hline Age & $60.2 \pm 9.8$ & $54.3 \pm 13.3$ & 0.043 \\
Gender (M/F) & $27 / 14$ & $32 / 18$ & 0.854 \\
LDL cholesterol (mmol/L) & $3.18 \pm 1.03$ & $2.49 \pm 0.73$ & 0.0013 \\
HDL cholesterol (mmol/L) & $1.14 \pm 0.29$ & $1.18 \pm 0.29$ & 0.596 \\
Triglycerides (mmol/L) & $1.44 \pm 0.64$ & $1.36 \pm 0.62$ & 0.568 \\
Uric acid (mmol/L) & $307.8 \pm 72.6$ & $283.4 \pm 80.2$ & 0.203 \\
\hline
\end{tabular}




\section{Cellular Physiology Cell Physiol Biochem 2017;42:729-742 \begin{tabular}{l|l|l} 
and BOI: 10.1159/000477890 & $\begin{array}{l}\text { C } 2017 \text { The Author(s). Published by S. Karger AG, Basel } \\
\text { www.karger.com/cpb }\end{array}$
\end{tabular}}

Wang et al.: Cholesterol and Colorectal Cancer

and migrated cells were fixed in methanol and stained with $0.1 \%$ crystal violet. The number of migrating cells was counted with a microscope in five representative fields. The test was repeated three times.

Sphere formation assay

A total of $1 \times 10^{3}$ cells/well were cultured in DMEM/F12 medium (Gibco) containing 2\% FBS, 20 $\mathrm{ng} / \mathrm{ml}$ epidermal growth factor, $10 \mathrm{ng} / \mathrm{ml}$ basic fibroblast growth factor, $4 \mathrm{mg} / \mathrm{ml}$ heparin, and $1.25 \mathrm{mg} /$ $\mathrm{ml}$ amphotericin B (all from Thermo scientific, Worcester, MA, USA) in ultralow attachment well plates (Corning) at $37^{\circ} \mathrm{C}$ for 14 days. Sphere formation (minimum 50 cells in a spheroid) was assessed after 14 days, by manual counting under an inverted microscope (Olympus CKX31).

RNA extraction and gene expression by $q P C R$

Total RNA was extracted using the Trizol (Thermo scientific) method according to the manufacturer's instructions. RNA quantity and quality were evaluated using a Nanodrop spectrophotometer (Thermo scientific). RNA was reverse-transcribed into cDNA using the Reverse Transcriptase M-MLV (Promega), and the expression of Sox2, Oct4, Nanog and Bmi 1 was measured using SYBR Green PCR Master Mix (Applied Biosystems, Foster City, CA, USA) on the Stepone plus system (Applied Biosystems). Gene expression levels were normalized to GAPDH expression.

The primers for quantitative real time PCR (qRT-PCR) analysis were listed as follows:

Sox 2-F 5'-GCA GAC TTC ACA TGT CCC AGC-3'

Sox 2-R 5'-CCG GGT TTT CTC CAT GCT GTT-3'

Oct 4-F 5'-TTC TCA GGG GGA CCA GTG TC-3'

Oct 4-R 5'-CCC ATT CCT AGA AGG GCA GG-3'

Nanog-F 5'-CCA GTG ACT TGG AGG CTG C-3'

Nanog-R 5'-AAG GAT TCA GCC AGT GTCC-3'

Bmi 1-F 5'-TGA CAA ATG CTG GAG AACTG-3'

Bmi 1-R 5'-AAG ATT GGT GGT TAC CGCT-3'

GAPDH-F 5'-CTG CCA ACG TGT CAG TGGTG-3'

GAPDH-R 5'-TCA GTG TAG CCC AGG ATGCC-3'

\section{Xenograft tumor growth assay}

Twenty 4-week old nude mice were randomly assigned to two groups: the standard chow diet group $(n=10)$ and the high fat diet (HFD) group $(n=10)$. Mice in the HFD group were treated with a dietary chow consisting of $60 \%$ kcal fat (D12079B) (Research Diets, New Brunswick, USA). The mice were treated with standard chow diet or HFD for 2 weeks, and then LoVo cells $\left(1 \times 10^{6}\right.$ for each mouse $)$ were injected into the backside of the five randomly selected mice for the HFD group and the standard chow group. The mice were killed and their weights were measured at week 4 , and tumor volumes of each mouse were measured weekly as length $\times$ width $^{2} / 2$. The blood LDL levels of the mice were assessed following the instruction manual of the cholesterol assay kit (Thermo scientific).

Tumorigenicity in AOM/DSS-treated mice

Thirty A/J mice were randomly divided into two groups and treated with either HFD or standard chow for two weeks. Following this, mice were treated with AOM by intraperitoneal injection at a dose of $0.2 \mathrm{mg}$ on the first day of week 1 . Dextran sulfate sodium (DSS) $(2 \mathrm{~g} / 100 \mathrm{ml})$ in drinking water was administered for one week at weeks 3, 6 and 9 (Fig. 6A). Mice were treated with either HFD or standard chow according to their group assignment, from week 1 to week 16 . We recorded the general health status of the mice every day, when conditions such as severe hematochezia or diarrhea, severe weight loss, inability to ambulate occurred, the mouse was considered to be moribund and was euthanized by cervical dislocation, and the mice survived at week 16 were also euthanized. Finally, a total of sixteen mice were euthanized at week 16, from which the intestinal lengths were measured and intestinal tissue samples were collected. The other fourteen mice were euthanized before week 16 because of moribund conditions. Data from these fourteen mice were excluded to ensure the reliability of the results. Intestinal tissue samples were processed and sectioned $(4 \mu \mathrm{m})$, stained with haematoxylin and eosin, and assessed under a light microscope by one researcher who was blinded to the treatments. Each section was evaluated for the presence of inflammatory lesions, tissue destruction, and tissue repair. 


\section{Cellular Physiology Cell Physiol Biochem 2017;42:729-742 \begin{tabular}{l|l} 
and Biochemistry Published online: June 09, 2017 & $\begin{array}{l}\text { (c) } 2017 \text { The Author(s). Published by S. Karger AG, Basel } \\
\text { www.karger.com/cpb }\end{array}$
\end{tabular}}

Wang et al.: Cholesterol and Colorectal Cancer

Immunohistochemistry

Anti-LDL Receptor antibody (ab30532) (Abcam, Cambridge, MA, USA) (1:100 dilution) was used to evaluate the abundance of LDL Receptor protein in the 90 CRC samples. Briefly, the extent of immunostaining was defined as 0 for $0 \%$ positive cells under the microscope, 1 for $1-25 \%$ positive cells, 2 for $26-50 \%$ positive cells, 3 for $51-75 \%$ positive cells and 4 for $\geq 76 \%$ positive cells. Intensity was scored as 0 for absence of staining, 1 for weak, 2 for moderate, and 3 for strong staining.

ROS assays

SW480, LoVo and RKO cell lines were treated with LDL $(100 \mu \mathrm{g} / \mathrm{ml})$ for $36 \mathrm{~h}$, followed by ROS measurement using dichloro-dihydro-fluorescein diacetate (DCFH-DA) (D6883) (Sigma, St Louis, MO, USA) according to the manufacturer's instructions. Briefly, cells were loaded with DCFH-DA (at a final concentration of $10 \mu \mathrm{M}$ for $30 \mathrm{~min}$ ), washed with ice cold Hanks Balanced Salt Solution, then observed under a Zeiss LSM710 laser confocal microscope (Carl Zeiss, Germany) equipped with Zen software to process the images.

Gene expression array analysis and GSEA

We used a Whole Human Genome Microarray (HOA 7.1) to obtain the expression profiles of each cell line treated with LDL or controls. After global normalization of the data across replicates, the differentially expressed genes (DEG) were identified as upregulated if the fold change was greater than or equal to 1.5 and as down regulated if the fold change was less than or equal to $-1.5(\mathrm{P}<0.05)$. KEGG pathway enrichment analysis was conducted for each group of DEGs by DAVID (https://david.ncifcrf.gov/) to identify and compare the enriched KEGG pathways.

\section{Western blot}

The DEGs were largely clustered in the p38 MAPK signaling pathway, thus we further assessed the levels of phosphorylated (p-p38) and p38 by western blot method. Total protein was extracted from cells lysed with the M-PER Mammalian Protein Extraction Reagent (Thermo Fisher Scientific, Rockford, USA). After blocking with 5\% non-fat milk in TBST for $60 \mathrm{~min}$, membranes were incubated with primary antibody dissolved in $5 \%$ bovine serum albumin in TBST overnight at $4^{\circ} \mathrm{C}$. The following primary antibodies were used: anti-human-p-p38 (1:2000, Cell Signaling Technology, Danvers, MA, USA), anti-human-p38 (1:2000, Cell Signaling Technology). Human GAPDH (1:5000, KangChen, Shanghai, China) was used as an internal reference.

\section{Statistical analysis}

All data are expressed as mean \pm Std. Statistical differences were measured using an unpaired twosided Student's t-test or one-way ANOVA for multiple comparisons when appropriate. Logistic analysis was used for the analysis of the association between LDL level and liver metastasis for CRC patients. $\mathrm{P}<0.05$ was defined as statistically significant. All data analysis was conducted with GraphPad Prism Software Version 5 (GraphPad, San Diego, CA).

\section{Results}

Elevated LDL levels in CRC patients with liver metastases

The role of cholesterol in cancer development appears to be controversial; higher serum cholesterol has been reported to be associated with cancer risk, while serum cholesterol has often been found to be decreased in advanced stage cancer patients. We included 41 CRC patients with liver metastases and 50 CRC patients without liver metastases in the current study, to assess the association between blood lipid levels and liver metastases in CRC patients, all of whom were newly diagnosed. The characteristics of

Fig. 1. LDL levels of CRC patients with liver metastases compared with CRC patients without liver metastases.






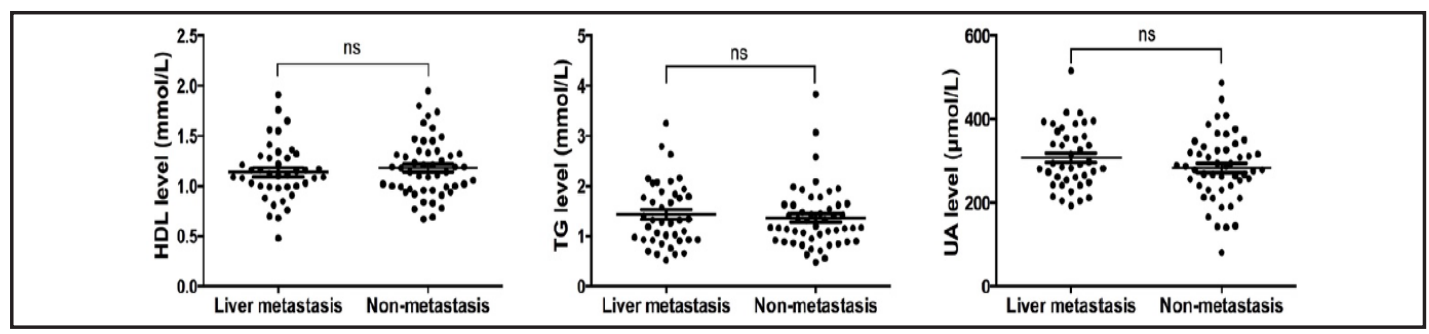

Fig. 2. HDL, triglycerides and uric acid levels of CRC patients with liver metastases compared with CRC patients without liver metastases.

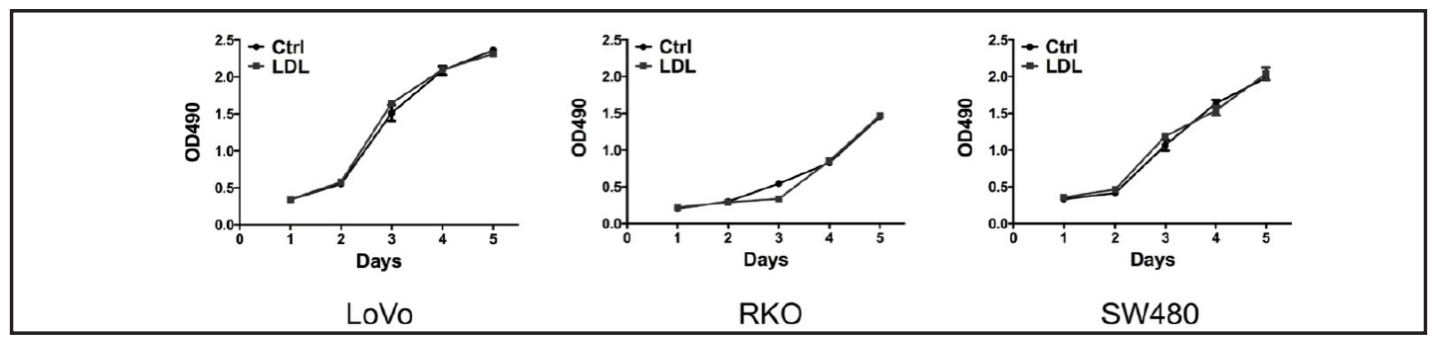

Fig. 3. Cell proliferation of LoVo, RKO and SW480 cells exposed to LDL and controls.

the enrolled patients are shown in Table 1. CRC patients with liver metastases were older compared to those without (60.2 vs. 54.3, $p=0.043$ ), while there was no gender difference between those two groups. As shown in Fig. 1, LDL levels in CRC patients with liver metastases (mean LDL level=3.18 $\mathrm{mmol} / \mathrm{L}$, std $=1.03$ ) were significantly higher than in those without liver metastases (mean LDL level $=2.49 \mathrm{mmol} / \mathrm{L}$, std $=0.73)(p=0.0013)$. Using logistic regression analysis, it was found that liver metastases were significantly associated with higher LDL levels (odds ratio, crude OR $=3.47,95 \%$ CI 1.41-8.54). Then multiple logistic regression analysis was conducted with age and LDL, which were significantly different between liver metastasis group and the control group, and the age-adjusted OR was 2.26 (95\% CI 1.33-3.85). No difference was observed in the levels of HDL cholesterol, triglycerides or uric acid between the liver metastases group and the non-liver metastases group (Fig. 2). These results indicate a possible role for LDL in CRC metastasis. However, the sample size was not large and the selection bias could not be ruled out though the CRC patients were consecutively enrolled, thus further large-scale study would be helpful to clarify this issue.

\section{LDL may promote CRC cell stemness in vitro}

SW480, LoVo and RKO cells were used to examine the biological functions of LDL in vitro. CRC cells were exposed to LDL at a level of $100 \mu \mathrm{g} / \mathrm{ml}$, and then cell proliferation, transwell migration, and sphere formation assays were performed. As shown in Fig. 3, there was no difference in the proliferation rate of cells exposed to LDL and the controls. However, LoVo and RKO cells exposed to LDL showed a significantly higher migration potential compared with controls (Fig. 4A and 4C). Moreover, LDL appeared to be positively associated with the number of spheres formed, for SW480 and LoVo cells (Fig. 4B and 4D). Expression of stemness-related genes was measured in CRC cells treated with LDL for 3 days and the controls using RT-qPCR, and it was found that LDL upregulated the expression of stemness genes including Sox2, Oct4, Nanog and Bmi 1 (Fig. 4E). Cancer stem cells play a crucial role in carcinogenesis and cancer recurrence, as well as metastasis and therapy resistance. Our results demonstrate that LDL promotes sphere formation and upregulates the expression of stemness genes, suggesting that LDL may enhance CRC cell stemness.

HFD promotes xenograft tumor growth

Administration of a HFD that was high in cholesterol was used to assess the effects of cholesterol on CRC tumorigenicity in vivo. Nude mice were randomly assigned into 
Fig. 4. Stem cell-like properties induced by LDL in CRC cells. (A) Representative photographs of cell migration assays of CRC cell lines treated with LDL, compared with controls. (B) Representative photographs of sphere formation of LoVo and SW480 cells treated with LDL, compared with controls. (C) Statistical analysis of cell migration assays. (D) Statistical analysis of sphere formation experiments. (E) RT-PCR results of stemness genes including Sox2, Oct4, Nanog and Bmi1 in LoVo and SW480 cells treated with LDL, compared with controls. * indicates $\mathrm{P}<0.05$.



B

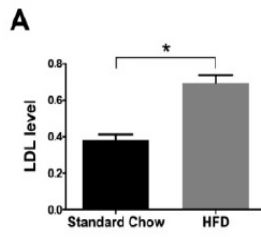

Non-xenograft

C

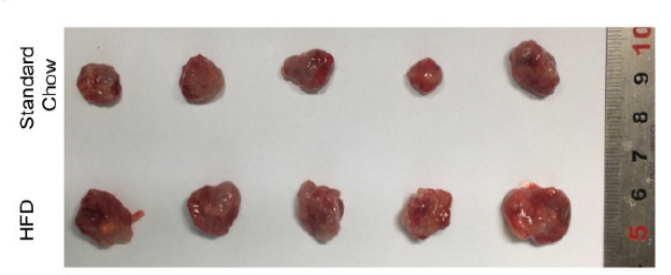

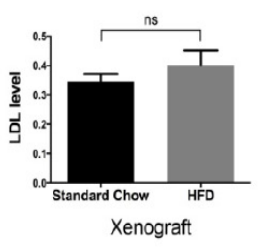

Xenograft

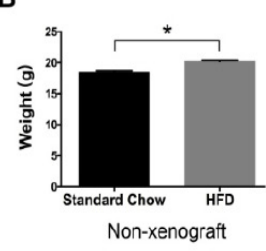

D
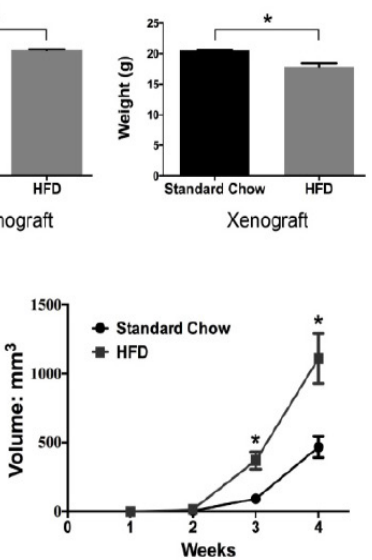

Fig. 5. HFD promotes xenograft tumor growth. (A) LDL levels of non-xenograft and xenograft mice treated with HFD or standard chow. (B) Weight of non-xenograft and xenograft mice treated with HFD or standard chow. (C) Representative images of xenograft tumor growth in vivo in mice treated with HFD compared with standard chow. (D) Xenograft tumor volume for mice treated with HFD and standard chow. * indicates $\mathrm{P}<$ 0.05 .

the standard chow diet group or the HFD group, with ten mice in each group. Then, five randomly selected mice from the standard chow diet group and five from the HFD group were injected with LoVo cells, while the remaining mice were used as the non-xenograft groups. As expected, HFD was associated with an increased blood LDL level $(0.694 \mathrm{mmol} / \mathrm{L}$ 
Fig. 6. HFD promotes intestinal inflammation in AOM/DSS-treated mice. (A) AOM/DSS treatment strategy for A/J mice. (B) Images of intestinal tissue from mice treated with HFD and standard chow. (C) Statistical analysis of intestinal length between mice treated with HFD and standard chow. (D) H\&E stain results of intestinal tissue of mice treated with HFD and standard chow. The red arrow indicates destruction of the epithelial architecture, with loss of crypts and epithelial integrity, submucosal edema, intense inflammatory cellular infiltration and lymphoid hyperplasia, dysplasia and nuclear hyperchromatism.
Fig. 7. LDLR expression in CRC patients. (A) Immunohistochemistry images of LDLR in CRC tissues and adjacent normal tissues. (B) Comparison of LDLR expression levels between CRC tissues and adjacent tissues. (C) Images displaying different degrees of LDLR expression levels $(-,+,++,+++)$. (D) LDLR expression levels in patients with different $\mathrm{N}$ stages. (E) LDLR expression levels in patients with different M stages.
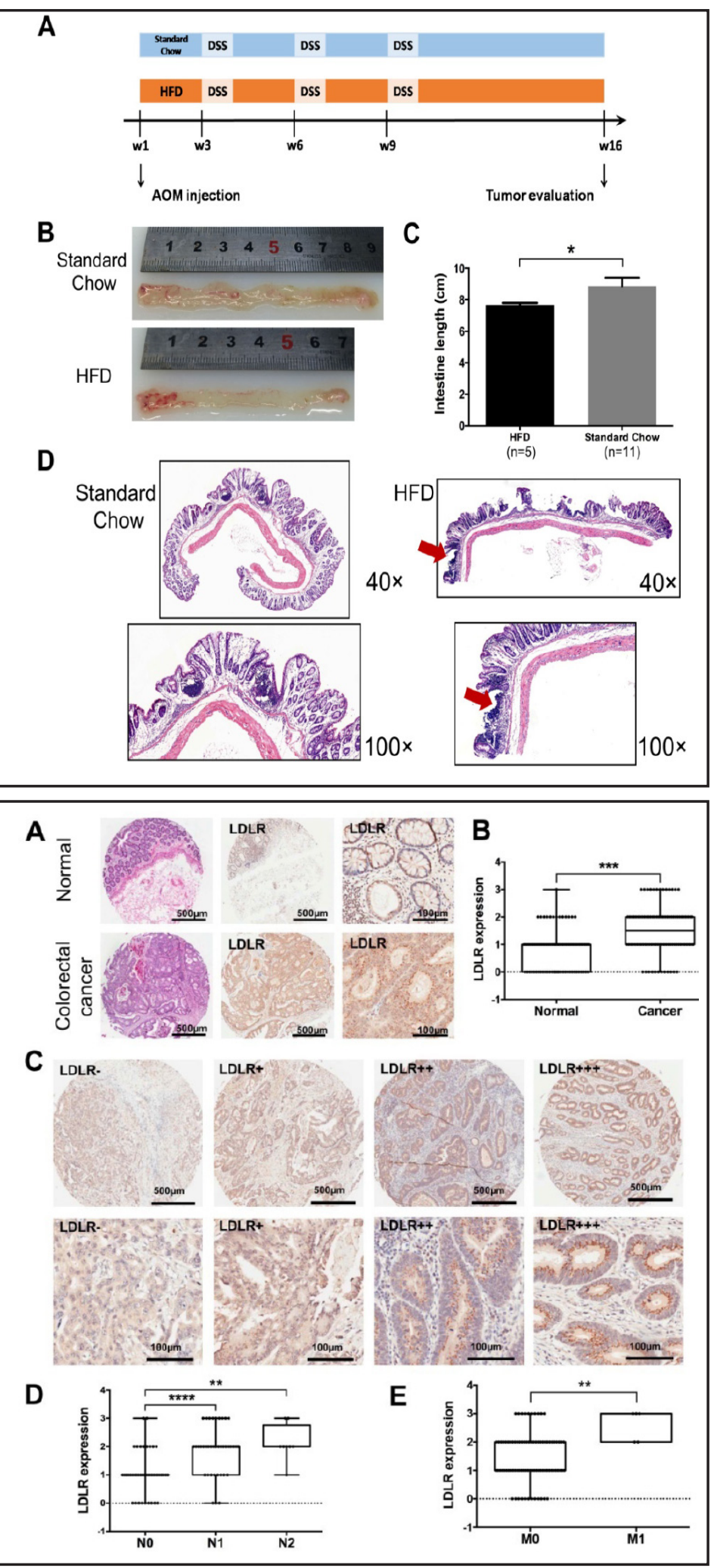

vs. $0.380 \mathrm{mmol} / \mathrm{L}, p=0.008$ ) compared with the standard chow group of non-xenograft mice. However, LDL levels measured in xenograft mice with HFD were similar to those in xenograft mice from the standard chow group $(0.402 \mathrm{mmol} / \mathrm{L}$ vs. $0.346 \mathrm{mmol} / \mathrm{L}, p=0.347)$ (Fig. 5A), indicating that xenografts might play a role in cholesterol levels. Moreover, non-xenograft 
Fig. 8. Association between LDLR expression levels and survival and T stage. (A) LDLR expression levels are not associated with CRC survival. (B) LDLR expression levels are not linked to T stage of CRC.


mice from the HFD group had higher weights than corresponding mice from the standard chow group (mean weight $=20.2 \mathrm{~g}$ vs $18.5 \mathrm{~g}, p=0.008$ ). In contrast, xenograft mice from the HFD group had lower weights than the corresponding mice from the standard chow group (mean weight $=17.9 \mathrm{~g}$ vs $20.5 \mathrm{~g}, p=0.008$ ) (Fig. 5B). Images of tumors excised from xenograft mice after 4 weeks and tumor volumes are shown in Fig. 5C and 5D, indicating that tumor volumes were significantly higher in mice that were administered a HFD diet compared with those who were fed standard chow. Thus, these findings suggest that HFD promotes tumor growth, while tumor growth may also have a negative role in cholesterol level.

\section{HFD promotes intestinal inflammation}

We next investigated the effects of HFD on CRC tumorigenesis in AOM/DSS-treated mice (Fig. 6A). The intestinal lengths of mice treated with HFD were significantly shorter than the mice fed with standard chow ( $p<0.05)$ (Fig. 6B and 6C), and a shorter intestinal length has been widely used as a marker of inflammation. As suggested in Fig. 6D, the intestines of mice treated with HFD showed destruction of the epithelial architecture, with loss of crypts and epithelial integrity, submucosal edema, intense inflammatory cellular infiltration and lymphoid hyperplasia, dysplasia and nuclear hyperchromatism (as indicated by the red arrow). The shorter intestinal lengths, inflammatory cellular infiltration, and nuclear hyperchromatism in HFD-treated mice indicate that HFD promotes intestinal inflammation, while the limited time (16 weeks) may partially explain why no obvious adenoma or tumor structure was found.

\section{LDLR expression levels are upregulated and associated with progression in CRC}

LDLR mediates the uptake of LDL from the circulation into cells, and over-production of LDLR has been observed to be an important mechanism for cancer cells to obtain more essential fatty acids [18]. The LDLR expression levels in cancer tissue and adjacent normal colorectal tissue from CRC patients were assessed using immunohistochemistry analysis. Figure 7A and 7C show representative images of different LDLR expression levels. LDLR expression levels were significantly higher in CRC tissues relative to adjacent normal colorectal tissues (Fig. 7B). We then sought to determine whether LDLR expression levels in CRC tissues were associated with cancer stages. Tumors from CRC patients with higher $\mathrm{N}$ or M stages demonstrated higher LDLR expression levels than those with lower stages (Fig. 7D and 7E); however, LDLR expression levels were not associated with T stages (Fig. 8B). We also found that LDLR expression levels were not associated with survival in CRC patients (Fig. 8A).

\section{LDL causes elevated ROS levels and activates the MAPK pathway}

Previous studies have indicated that HFD contributed to excessive production of reactive ROS [13] and, therefore, we investigated the effects of LDL on ROS levels of SW480, LoVo and RKO, using DCFH-DA fluorescence analysis. The results showed that all types of CRC cells treated with LDL exhibited significantly higher ROS levels compared with controls (Fig. 9A and 9B). Furthermore, we used a Whole Human Genome Microarray to obtain the expression profiles of each cell line, and the number of DEGs identified between the SW480, LoVo and RKO cells treated with LDL and their controls, was 210, 268 and 733, respectively. 


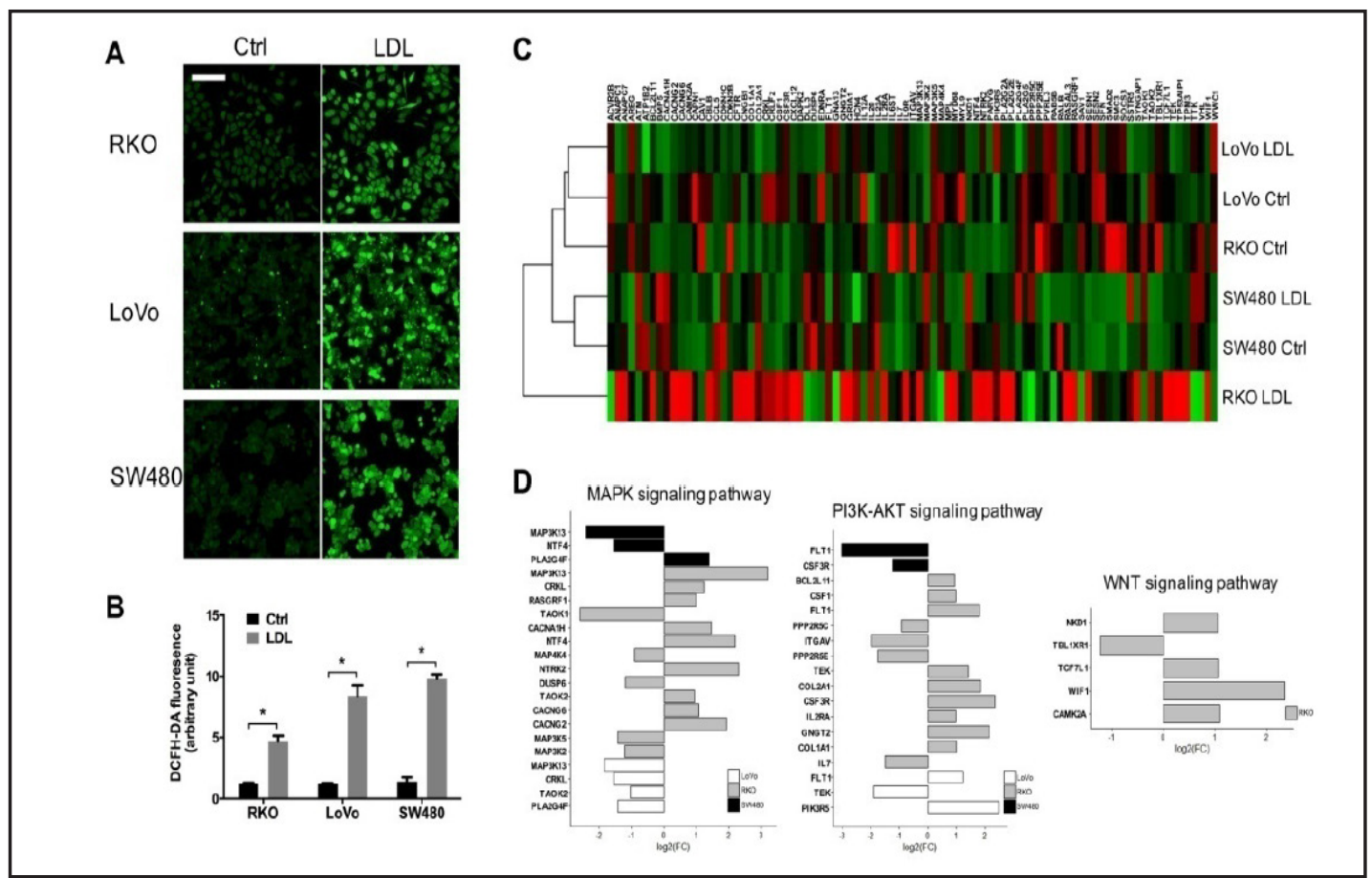

Fig. 9. LDL elevates ROS levels and activates the MAPK signaling pathway in CRC cells. (A) Representative images of DCFH-DA fluorescence analysis suggesting that LDL elevates ROS levels in RKO, LoVo and SW480 CRC cells. (B) DCFH-DA fluorescence levels in CRC cells. (C) Images of the whole human genome microarray for CRC cells treated with LDL and controls. (D) Differentially expressed genes (DEGs) for CRC cells treated with LDL compared with controls. Fold change of expression level $\geq 1.5$ indicates upregulated DEGs and fold change $<1.5$ indicates downregulated DEGs.

KEGG pathway enrichment analysis was performed for each group of DEGs using DAVID (https://david.ncifcrf.gov/), from which 87 DEGs were found to participate in the enriched pathways. The result of unsupervised hierarchical clustering analysis from the overlapped 87 DEGs is shown in Fig. 9C. Besides these overlapping DEGs, we were also interested in the DEGs that participated in some important signaling pathways, such as the MAPK, WNT, PI3K-AKT and apoptotic

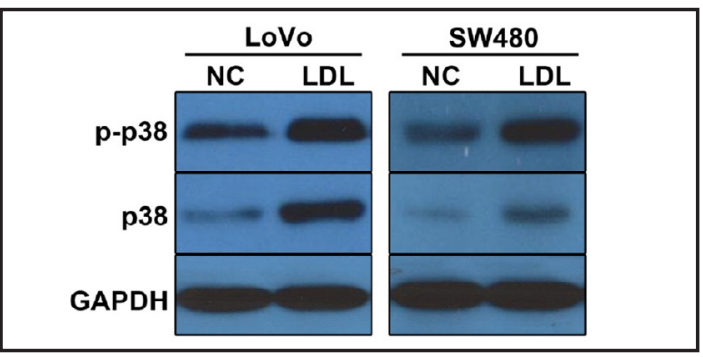

Fig. 10. LDL promoted p38 and phosphorylated p38 levels in LoVo and SW480 cells.

pathways. The fold change values of DEGs that participated in these pathways are shown in Fig. 9D. Importantly, the DEGs were largely clustered in the MAPK signaling pathway, especially the p38 MAPK pathway. By Western blot method, we observed that LDL elevates p-p38 and p38 levels in CRC cells (Fig. 10), which further confirmed the finding.

\section{Discussion}

Epidemiologic studies have suggested a possible role for obesity and cholesterol in cancer. A substantial body of evidence supports an association between obesity and various cancers, including CRC [19]. Elevated serum cholesterol levels were shown to be linked to a higher risk of developing certain cancers, and higher cholesterol levels may also increase cancer recurrence [20,21]. Moreover, statins use in patients with cancer is associated with a reduced cancer-related mortality [22]. Cholesterol metabolites have also been associated 
with the development of various cancers in animal studies [23, 24]. HFD was also observed to accelerate cancer progression and reduce survival rates in mice [25]. However, only a few studies have evaluated the effects of blood cholesterol levels on cancer metastasis, and the underlying mechanisms remain unclear. Herein, we performed a cohort study with CRC patients, and found significantly higher LDL levels in CRC patients with liver metastases than in those without, indicating that cholesterol may play a role in CRC metastasis. We also assessed the expression levels of LDLR and observed increased LDLR expression in CRC tissues especially in CRC patients with advanced $\mathrm{N}$ and M stages. LDL and HFD increased CRC cell growth and progression both in vitro and in vivo, and LDL caused elevated ROS levels and MAPK pathway activation.

Cholesterol has been observed to accumulate, generally, in various malignant tissues; however, although studies have suggested that cholesterol plays an important role in cancer progression [5,6], advanced stage cancer patients tend to have lower cholesterol levels. To explain this inconsistency, a cohort study was performed enrolling only newly diagnosed CRC patients who had not undergone surgery, radiotherapy or chemotherapy and, therefore, mainly displayed normal nutritional compositions. We found that serum LDL levels in CRC patients with liver metastases were significantly higher than in those without liver metastases. Further studies were conducted using animal models, from which it was demonstrated that tumor volumes of HFD-treated xenograft mice were significantly larger than those from the standard chow group, while blood LDL levels were similar in the two groups. We observed similar LDL levels between xenograft mice treated with HFD and standard chow, and moreover, the weights of xenograft mice from the HFD group were lower than the mice from the standard chow group. Considering that HFD increased blood LDL levels and weights in non-xenograft mice, it might be speculated that tumor growth also has a negative role in cholesterol level and weight. In fact, serum lipids levels and weights of cancer patients at advanced stages might decrease because of poor nutrition status [7]. LDLR expression level was upregulated in CRC tissue relative to adjacent normal tissue, and was associated with advanced $\mathrm{N}$ and $\mathrm{M}$ stages; since LDLR is essential for transporting LDL from the blood into cells, we speculated that cholesterol levels would effect CRC metastasis. LDL was observed to promote migration of CRC cells, while no association between LDL and CRC cell proliferation was found. Moreover, LDL was observed to promote sphere formation of CRC cells in vitro, and LDL also upregulated the expression of stemness genes including Sox2, Oct4, Nanog and Bmi 1, indicating possible induction of stem cell-like properties by LDL. Cancer stem cells can be resistant to therapy and, thus, play a vital role in cancer initiation, relapse and metastasis. This is consistent with previous studies suggesting that HFD promotes colonic stem cell expansion and tumor initiation $[10,11]$.

In the present study, we observed that LDL significantly elevates ROS levels in CRC cells. ROS are produced within cells through oxygen metabolism and act as important signaling molecules in the processes of cell proliferation, survival, and apoptosis [26]. Intracellular ROS are mainly synthetized in the mitochondria and therein enhance the accumulation of ROS, oxidization of proteins, lipids and DNA, and lead to redox imbalance and oxidative stress [27]. In addition, ROS induce secretion of pro- or anti-inflammatory molecules and thus play an important role in inflammation during cancer development [28]. Oxidative stress can promote inflammation, and excess inflammation can, in turn, increase oxidative stress, causing damage to cells and tissues [29]. AOM/DSS-treated mice were used to explore the effects of HFD on intestinal tumorigenesis, and the results suggested that intestinal lengths, widely used as a marker of inflammation in inflammatory bowel disease animal models, were shorter in mice treated with HFD. Damage to the epithelial architecture was also observed in HFD-treated mice, as well as intense inflammatory cellular infiltration and lymphoid hyperplasia, and nuclear hyperchromatism. However, no significant adenoma or tumor structures were found, possibly because of the limited treatment time (16 weeks), so further studies with longer treatment times would be helpful to clarify this issue. We observed that HFD enhanced intestinal inflammation, which is strongly associated with cancer initiation. 
The underlying molecular mechanisms were also explored by microarray; 87 differentially expressed genes (DEGs) were found between three CRC cell lines treated with LDL and the controls. These genes were clustered in some important signaling pathways, such as MAPK, WNT and PI3K-AKT. We also observed increased levels of p38 and phosphorylated p38 by western blot, and further confirmed the activation of MAPK pathway. This is consistent with previous reports indicating that LDL and VLDL could activate the MAPK pathway [14]. MAPK pathway activation has been widely recognized to play a vital role in the regulation of gene expression, mitosis, metabolism, cell proliferation, apoptosis and cellular movement [30]. There are four classes of MAPK signaling cascades with functions in mammary epithelial cells and involvement in tumor progression, including extracellularregulated kinase (ERK)1/2, c-Jun N-terminal kinase (JNK), p38 MAPK and ERK5 [31]. ERK1/2 primarily functions in cell proliferation [32], while activation of JNK and p38 MAPK mainly promote cell apoptosis [33]. Moreover, MAPKs have been found to be important signaling cascades in the processes of epithelial-mesenchymal transition (EMT) and cell migration, which are mediated by PKC, TGF-beta/Smad [34] and integrin-mediated signaling [35]. EMT is the first step in metastasis, which is characterized by the loss of epithelial polarity and cell-cell adhesion and is a precursor to increased cell mobility. MAPKs are involved in cell migration and invasion, partially through regulation of focal adhesion kinase (FAK) and matrix metalloproteinases (MMPs) [36-38]. Previous studies have indicated that ROS could regulate PI3K/Akt and MAPK signaling pathways and thus be involved in apoptosis [39]. ROS accumulation could inactivate JNK phosphatases and TNF-mediated necrosis, resulting in prolonged activation of JNK [40]. ROS was also shown to mediate MAPK activation in EMT [41]. Moreover, ROS-activated MAPK signaling was shown to induce migration of smooth muscle cells [42]. Thus, it could be speculated that LDL enhanced tumor progression via a ROS-activated MAPK pathway. Further experiments, including qRT-PCR and western blot should be conducted to confirm direct activation of MAPK pathway.

In summary, the present study indicates that LDL enhances intestinal inflammation and CRC progression, possibly via activation of ROS and pathways including MAPK pathway. Future research is needed to further explore the effect of LDL on intestinal tumorigenicity, meanwhile, the pathways investigated in the current study should also be confirmed. Our study highlights the effects of cholesterol on CRC progression, providing a better understanding of the mechanisms regulating CRC and identifying a potential target for the treatment of CRC. Cholesterol-lowering strategies, including reduction of body weight and statins, may be helpful in reducing CRC risk, and statins could also be evaluated as a potential adjunctive therapy for CRC.

\section{Acknowledgements}

This work was supported by grants from the National Natural Science Foundation of China (No. 81402324), Zhejiang Provincial Natural Science Foundation of China (No. LY14H160027, No. LY16H160019).

\section{Disclosure Statement}

The authors declare no competing financial interests.

\section{References}

1 Torre LA, Bray F, Siegel RL, Ferlay J, Lortet-Tieulent J, Jemal A: Global cancer statistics, 2012. CA Cancer J Clin 2015;65:87-108. 


\section{Cellular Physiology Cell Physiol Biochem 2017;42:729-742 \begin{tabular}{l|l} 
DOI: 10.1159/000477890 & O 2017 The Author(s). Published by S. Karger AG, Basel \\
www.karger.com/cpb
\end{tabular} \\ Wang et al.: Cholesterol and Colorectal Cancer}

-2 Qi J, Yu Y, Akilli Ozturk O, Holland JD, Besser D, Fritzmann J, Wulf-Goldenberg A, Eckert K, Fichtner I, Birchmeier W: New Wnt/beta-catenin target genes promote experimental metastasis and migration of colorectal cancer cells through different signals. Gut DOI:10.1136/gutjnl-2014-307900.

-3 Lochhead P, Chan AT, Giovannucci E, Fuchs CS, Wu K, Nishihara R, O'Brien M, Ogino S: Progress and opportunities in molecular pathological epidemiology of colorectal premalignant lesions. Am J Gastroenterol 2014;109:1205-1214.

-4 Yang MH, Rampal S, Sung J, Choi YH, Son HJ, Lee JH, Kim YH, Chang DK, Rhee PL, Kim JJ, Rhee JC, Chun HK, Guallar E, Cho J: The association of serum lipids with colorectal adenomas. Am J Gastroenterol 2013;108:833-841.

-5 Freeman MR, Solomon KR: Cholesterol and prostate cancer. J Cell Biochem 2004;91:54-69.

-6 Llaverias G, Danilo C, Mercier I, Daumer K, Capozza F, Williams TM, Sotgia F, Lisanti MP, Frank PG: Role of cholesterol in the development and progression of breast cancer. Am J Pathol 2011;178:402-412.

7 Han JE, Lee JY, Bu SY: The Level of Serum Cholesterol is Negatively Associated with Lean Body Mass in Korean non-Diabetic Cancer Patients. Clin Nutr Res 2016;5:126-136.

-8 Cangelosi AL, Yilmaz OH: High fat diet and stem cells: Linking diet to intestinal tumor formation. Cell Cycle 2016;15:1657-1658.

9 Mihaylova MM, Sabatini DM, Yilmaz OH: Dietary and metabolic control of stem cell function in physiology and cancer. Cell Stem Cell 2014;14:292-305.

10 Beyaz S, Mana MD, Roper J, Kedrin D, Saadatpour A, Hong SJ, Bauer-Rowe KE, Xifaras ME, Akkad A, Arias E, Pinello L, Katz Y, Shinagare S, Abu-Remaileh M, Mihaylova MM, Lamming DW, Dogum R, Guo G, Bell GW, Selig M, Nielsen GP, Gupta N, Ferrone CR, Deshpande V, Yuan GC, Orkin SH, Sabatini DM, Yilmaz OH: High-fat diet enhances stemness and tumorigenicity of intestinal progenitors. Nature 2016;531:53-58.

11 DeClercq V, McMurray DN, Chapkin RS: Obesity promotes colonic stem cell expansion during cancer initiation. Cancer Lett 2015;369:336-343.

12 Song L, Li Y: The Role of Stem Cell DNA Methylation in Colorectal Carcinogenesis. Stem Cell Rev DOI:10.1007/s12015-016-9672-6.

13 Ding C, Zhao Y, Shi X, Zhang N, Zu G, Li Z, Zhou J, Gao D, Lv L, Tian X, Yao J: New insights into salvianolic acid A action: Regulation of the TXNIP/NLRP3 and TXNIP/ChREBP pathways ameliorates HFD-induced NAFLD in rats. Sci Rep 2016;6:28734.

14 Menard JA, Christianson HC, Kucharzewska P, Bourseau-Guilmain E, Svensson KJ, Lindqvist E, Indira Chandran V, Kjellen L, Welinder C, Bengzon J, Johansson MC, Belting M: Metastasis Stimulation by Hypoxia and Acidosis-Induced Extracellular Lipid Uptake Is Mediated by Proteoglycan-Dependent Endocytosis. Cancer Res 2016;76:4828-4840.

15 Poillet-Perez L, Despouy G, Delage-Mourroux R, Boyer-Guittaut M: Interplay between ROS and autophagy in cancer cells, from tumor initiation to cancer therapy. Redox Biol 2015;4:184-192.

16 Szatrowski TP, Nathan CF: Production of large amounts of hydrogen peroxide by human tumor cells. Cancer Res 1991;51:794-798.

17 Sun B, Hu X, Liu G, Ma B, Xu Y, Yang T, Shi J, Yang F, Li H, Zhang L, Zhao Y: Phosphatase Wip1 negatively regulates neutrophil migration and inflammation. J Immunol 2014;192:1184-1195.

-18 Chen Y, Hughes-Fulford M: Human prostate cancer cells lack feedback regulation of low-density lipoprotein receptor and its regulator, SREBP2. Int J Cancer 2001;91:41-45.

$\rightarrow 19$ Klil-Drori AJ, Azoulay L, Pollak MN: Cancer, obesity, diabetes, and antidiabetic drugs: is the fog clearing? Nat Rev Clin Oncol DOI:10.1038/nrclinonc.2016.120.

20 Allott EH, Howard LE, Cooperberg MR, Kane CJ, Aronson WJ, Terris MK, Amling CL, Freedland SJ: Serum lipid profile and risk of prostate cancer recurrence: Results from the SEARCH database. Cancer Epidemiol Biomarkers Prev 2014;23:2349-2356.

21 Shafique K, McLoone P, Qureshi K, Leung H, Hart C, Morrison DS: Cholesterol and the risk of grade-specific prostate cancer incidence: evidence from two large prospective cohort studies with up to 37 years' follow up. BMC Cancer 2012;12:25.

22 Nielsen SF, Nordestgaard BG, Bojesen SE: Statin use and reduced cancer-related mortality. N Engl J Med 2012;367:1792-1802.

23 McDonnell DP, Park S, Goulet MT, Jasper J, Wardell SE, Chang CY, Norris JD, Guyton JR, Nelson ER: Obesity, cholesterol metabolism, and breast cancer pathogenesis. Cancer Res 2014;74:4976-4982. 


\section{Cellular Physiology Cell Physiol Biochem 2017;42:729-742 \begin{tabular}{l|l} 
DOI: 10.1159/000477890 & and Biochemistry \\
Published onlne: June 09, 2017 & $\begin{array}{l}\text { O 2017 The Author(s). Published by S. Karger AG, Basel } \\
\text { www.karger.com/cpb }\end{array}$
\end{tabular}}

Wang et al.: Cholesterol and Colorectal Cancer

24 Lin CY, Huo C, Kuo LK, Hiipakka RA, Jones RB, Lin HP, Hung Y, Su LC, Tseng JC, Kuo YY, Wang YL, Fukui Y, Kao YH, Kokontis JM, Yeh CC, Chen L, Yang SD, Fu HH, Chen YW, Tsai KK, Chang JY, Chuu CP: Cholestane-3beta, 5alpha, 6beta-triol suppresses proliferation, migration, and invasion of human prostate cancer cells. PLoS One 2013;8:e65734.

25 Cho HJ, Kwon GT, Park H, Song H, Lee KW, Kim JI, Park JH: A high-fat diet containing lard accelerates prostate cancer progression and reduces survival rate in mice: possible contribution of adipose tissuederived cytokines. Nutrients 2015;7:2539-2561.

26 Gough DR, Cotter TG: Hydrogen peroxide: a Jekyll and Hyde signalling molecule. Cell Death Dis 2011;2:e213.

-27 Fruehauf JP, Meyskens FL, Jr.: Reactive oxygen species: a breath of life or death? Clin Cancer Res 2007;13:789-794.

-28 Vendramini-Costa DB, Carvalho JE: Molecular link mechanisms between inflammation and cancer. Curr Pharm Des 2012;18:3831-3852.

29 Rimessi A, Previati M, Nigro F, Wieckowski MR, Pinton P: Mitochondrial reactive oxygen species and inflammation: Molecular mechanisms, diseases and promising therapies. Int J Biochem Cell Biol DOI:10.1016/j.biocel.2016.06.015.

30 Su B, Karin M: Mitogen-activated protein kinase cascades and regulation of gene expression. Curr Opin Immunol 1996;8:402-411.

-31 Whyte J, Bergin O, Bianchi A, McNally S, Martin F: Key signalling nodes in mammary gland development and cancer. Mitogen-activated protein kinase signalling in experimental models of breast cancer progression and in mammary gland development. Breast Cancer Res 2009;11:209.

-32 Roovers K, Assoian RK: Integrating the MAP kinase signal into the G1 phase cell cycle machinery. Bioessays 2000;22:818-826.

-33 Li X, Wang K, Ren Y, Zhang L, Tang XJ, Zhang HM, Zhao CQ Liu PJ, Zhang JM, He JJ: MAPK signaling mediates sinomenine hydrochloride-induced human breast cancer cell death via both reactive oxygen speciesdependent and -independent pathways: an in vitro and in vivo study. Cell Death Dis 2014;5:e1356.

-34 Javelaud D, Mauviel A: Crosstalk mechanisms between the mitogen-activated protein kinase pathways and Smad signaling downstream of TGF-beta: implications for carcinogenesis. Oncogene 2005;24:5742-5750.

35 Wu WS: The signaling mechanism of ROS in tumor progression. Cancer Metastasis Rev 2006;25:695-705.

-36 McFadden DG, Vernon A, Santiago PM, Martinez-McFaline R, Bhutkar A, Crowley DM, McMahon M, Sadow PM, Jacks T: p53 constrains progression to anaplastic thyroid carcinoma in a Braf-mutant mouse model of papillary thyroid cancer. Proc Natl Acad Sci USA 2014;111:E1600-1609.

37 Gao X, Balan V, Tai G, Raz A: Galectin-3 induces cell migration via a calcium-sensitive MAPK/ERK1/2 pathway. Oncotarget 2014;5:2077-2084.

-38 Wang H, An H, Wang B, Liao Q, Li W, Jin X, Cui S, Zhang Y, Ding Y, Zhao L: miR-133a represses tumour growth and metastasis in colorectal cancer by targeting LIM and SH3 protein 1 and inhibiting the MAPK pathway. Eur J Cancer 2013;49:3924-3935.

39 Wang J, Yuan L, Xiao H, Xiao C, Wang Y, Liu X: Momordin Ic induces HepG2 cell apoptosis through MAPK and PI3K/Akt-mediated mitochondrial pathways. Apoptosis 2013;18:751-765.

$\rightarrow 40$ Seki E, Brenner DA, Karin M: A liver full of JNK: signaling in regulation of cell function and disease pathogenesis, and clinical approaches. Gastroenterology 2012;143:307-320.

-41 Rhyu DY, Yang Y, Ha H, Lee GT, Song JS, Uh ST, Lee HB: Role of reactive oxygen species in TGF-beta1-induced mitogen-activated protein kinase activation and epithelial-mesenchymal transition in renal tubular epithelial cells. J Am Soc Nephrol 2005;16:667-675.

42 Lo IC, Shih JM, Jiang MJ: Reactive oxygen species and ERK 1/2 mediate monocyte chemotactic protein-1stimulated smooth muscle cell migration. J Biomed Sci 2005;12:377-388. 